\title{
THE ASYMPTOTIC EXPANSIONS FOR THE ODD PERIODIC MATHIEU FUNCTIONS
}

BY

\section{GERTRUDE BLANCH}

1. Background. Mathieu's equation

$$
y^{\prime \prime}+(a-2 q \cos 2 x) y=0
$$

admits of solutions of period $\pi$ and $2 \pi$ for characteristic values $a(q)$. If $q$ is positive and large there is the well-known expansion [1] in terms of reciprocal powers of $q^{1 / 2}$, which gives good results for small angles $x$. For angles close to $90^{\circ} \mathrm{Sips}$ [5] obtained a remarkably good expansion in terms of parabolic cylinder functions for the even solutions $c e_{n}(x, q)$, namely

$$
c e_{n}(x, q)=C_{n} \sum_{k=-\infty}^{\infty} g_{2 k} D_{n+2 k}(\alpha)
$$

where

$$
\begin{aligned}
\alpha & =2 q^{1 / 4} \cos x, \\
D_{m}(\alpha) & =(-1)^{m} e^{(1 / 4) \alpha^{2}} \frac{d^{m}}{d \alpha^{m}} e^{(-1 / 2) \alpha^{2}},
\end{aligned}
$$

and the $g_{2 k}$ involve reciprocal powers of $q^{1 / 2}$. He also gave the asymptotic form of the normalization factor $C_{n}$ which is appropriate for Ince's( $\left.{ }^{1}\right)$ normalization [2] namely

$$
\int_{0}^{\pi}\left[c e_{n}(x, q)\right]^{2} d x=\int_{0}^{\pi}\left[s e_{n}(x, q)\right]^{2} d x=\frac{1}{2} \pi .
$$

Sips argued that the odd periodic function $s_{n+1}(x, q)$ has precisely the same expansion as $c e_{n}(x, q)$ even though $s e_{n+1}(x, q)$ vanishes at $x=0$, because $\left|c e_{2 n}(0, q) / c e_{2 n}(\pi / 2, q)\right|$ and $\left|c e_{2 n+1}(0, q) / c e_{2 n+1}^{\prime}(\pi / 2, q)\right|$ are exponentially small as $q$ approaches infinity. Moreover, both $c e_{n}(x, q)$ and $s e_{n+1}(x, q)$ have similar behavior at $x=\pi / 2$.

Meixner and Schäfke [3] proved that a solution of the form

$$
\operatorname{se}_{n}(x, q)=\sin x \sum g_{2 k} D_{n+2 k}(\alpha)
$$

is an asymptotic expansion for $s_{n}(x, q)$; their development is limited to the leading term only (i.e. $O q^{-1 / 2}$ ). In the same chapter, the authors later give the equation

Received by the editors February 23, 1960.

(1) Sips actually used Goldstein's normalization for $c e_{0}(x, q)$ which differs from (1.04) by a factor of $2^{1 / 2}$. 


$$
s e_{n+1}(x, q) \cong \sin x c e_{n}(x, q) .
$$

listing the results of $\operatorname{Sips}$ for $c e_{n}(x, q)$ to terms in $1 / q$. It will be shown in $\S 2$ that (1.06) is correct in the leading term only and that the correct relations between $s e_{n+1}(x, q)$ and $c e_{n}(x, q)$, for a form of type (1.05) are

$$
\begin{gathered}
c e_{n}(x, q)=C_{n}\left[Z_{0, n}+Z_{1, n}\right], \\
s e_{n+1}(x, q)=S_{n}\left[Z_{0, n}-Z_{1, n}\right] \sin x
\end{gathered}
$$

where

$$
\begin{aligned}
& Z_{0, n}=\sum g_{4 k} D_{n+4 k}(\alpha), \\
& Z_{1, n}=\sum g_{4 k+2} D_{n+4 k+2}(\alpha) .
\end{aligned}
$$

The coefficients $g_{m}$ involve reciprocal powers of $q^{1 / 2}$, and their precise form is given explicitly in $\$ 3$, along with some examples. It is also shown that (1.08) is asymptotically equivalent to (1.07), for $0<x<\pi$. Equation (1.06) gives results that are considerably worse than either (1.07) or (1.08).

The most important formulas are summarized in $\$ 3$, in view of some misprints that appear in the scattered literature relating to them.

2. Derivation of expansion for $s_{n+1}(x, q)$. If (1.02) is substituted into (1.00), one obtains

$$
\left(1-\frac{\alpha^{2}}{4 q^{1 / 2}}\right) \frac{d^{2} y}{d \alpha^{2}}-\frac{\alpha}{4 q^{1 / 2}} \frac{d y}{d \alpha}+\left(A-\frac{1}{4} \alpha^{2}\right) y=0
$$

where

$$
A=(a+2 q) / 4 q^{1 / 2} .
$$

It is well known that for large values of $q$

$$
A=n+\frac{1}{2}-\frac{d_{1}}{4 q^{1 / 2}}-\frac{d_{2}}{\left(4 q^{1 / 2}\right)^{2}}-\cdots
$$

where the $d_{j}$ depend on $n$. (These $d_{j}$ are in fact recovered from the developments that follow.) Let

$$
y=\sin x U(\alpha) .
$$

The differential equation for $U(\alpha)$ becomes

$$
\left(1-\frac{\alpha^{2}}{4 q^{1 / 2}}\right) \frac{d^{2} U}{d \alpha^{2}}-\frac{3 \alpha}{4 q^{1 / 2}} \frac{d U}{d \alpha}+\left(-\frac{1}{4 q^{1 / 2}}+A-\frac{1}{4} \alpha^{2}\right) U=0
$$

Both (2.00) and (2.03) are of the form

$$
\left(1-\frac{\alpha^{2}}{4 q^{1 / 2}}\right) \frac{d^{2} W}{d \alpha^{2}}-\frac{\tau_{1} \alpha}{4 q^{1 / 2}} \frac{d W}{d \alpha}+\left(A-\frac{1}{4} \alpha^{2}-\frac{\tau_{2}}{4 q^{1 / 2}}\right) W=0
$$


The parameters of interest are:

Case 1, giving rise to form (2.00):

$$
\tau_{1}=1 ; \quad \tau_{2}=0 .
$$

Case 2, giving rise to (2.03):

$$
\tau_{1}=3 ; \quad \tau_{2}=1 .
$$

Define

$$
Y_{0}=D_{n}(\alpha)
$$

Thus $Y_{0}$ satisfies

$$
Y_{0}^{\prime \prime}+\left(n+\frac{1}{2}-\frac{1}{4} \alpha^{2}\right) Y_{0}=0
$$

Next define $Y_{1}$ from the differential equation

(2.071) $Y_{1}^{\prime \prime}+\left(n+\frac{1}{2}-\frac{1}{4} \alpha^{2}\right) Y_{1}=d_{1} Y_{0}+\left(\alpha^{2} Y_{0}^{\prime \prime}+\tau_{1} \alpha Y_{0}^{\prime}+\tau_{2} Y_{0}\right)$

where $d_{1}$ is to be determined. In general, if $Y_{0}, Y_{1}, \cdots, Y_{j-1}$ are known, define

$$
\begin{aligned}
& Y_{j}^{\prime \prime}+\left(n+\frac{1}{2}-\frac{1}{4} \alpha^{2}\right) Y_{j} \\
& =\left(Y_{0} d_{j}+Y_{1} d_{j-1}+\cdots+Y_{j-1} d_{1}\right)+\alpha^{2} Y_{j-1}^{\prime \prime}+\tau_{1} \alpha Y_{j-1}^{\prime}+\tau_{2} Y_{j-1} .
\end{aligned}
$$

Finally, let

$$
W_{j}=Y_{0}+\frac{Y_{1}}{4 q^{1 / 2}}+\cdots+\frac{Y_{j}}{\left(4 q^{1 / 2}\right)^{j}} .
$$

It can be verified that $W_{j}$ satisfies

$$
\left(1-\frac{\alpha^{2}}{4 q^{1 / 2}}\right) W_{j}^{\prime \prime}-\frac{\tau_{1} \alpha}{4 q^{1 / 2}} W_{j}^{\prime}+\left(A-\frac{1}{4} \alpha^{2}-\frac{\tau_{2}}{4 q^{1 / 2}}\right) W_{j}=\phi(\alpha, q)
$$

where

$$
\phi(\alpha, q)=O q^{(-1 / 2)(j+1)} .
$$

To solve for the $Y_{k}$, let

$$
Y_{k}=\sum_{p=-2 k}^{2 k} c_{n+2 p, j} D_{n+2 p}(\alpha)
$$

and determine the coefficients $c_{m}$ so as to satisfy (2.072). So far, this is precisely the method of Sips, except that it is applied to the more general 
equation (2.04). Two well known properties of the functions $D_{n}(\alpha)$ will be used:

$$
\begin{aligned}
& D_{n+2 p}^{\prime \prime}(\alpha)+\left(n+\frac{1}{2}-\frac{1}{4} \alpha^{2}\right) D_{n+2 p}(\alpha)=-2 p D_{n+2 p}(\alpha) \\
& \alpha^{2} D_{m}^{\prime \prime}(\alpha)+\tau_{1} \alpha D_{m}^{\prime}(\alpha)+\tau_{2} D_{m}(\alpha) \\
& \quad=\rho_{4} D_{m+4}(\alpha)+\rho_{0, m} D_{m}(\alpha)+\rho_{-2, m-2} D_{m-2}(\alpha)+\rho_{-4, m-4} D_{m-4}(\alpha),
\end{aligned}
$$

$m=n+2 p$, and

$$
\begin{aligned}
\rho_{4} & =\frac{1}{4} ; \quad \rho_{2}=\left(1-\frac{1}{2} \tau_{1}\right) ; \\
\rho_{0, n+2 p} & =\left[-\frac{1}{2}(n+2 p)^{2}-\frac{1}{2}(n+2 p)+\frac{1}{4}-\frac{1}{2} \tau_{1}+\tau_{2}\right] \\
\rho_{-2, n+2 p-2} & =\left(-2+\tau_{1}\right)\left(\begin{array}{c}
n+2 p \\
2
\end{array}\right) ; \quad \rho_{-4, n+2 p-4}=6\left(\begin{array}{c}
n+2 p \\
4
\end{array}\right) .
\end{aligned}
$$

Where no ambiguity is likely to arise, the symbol $D_{k}$ will be used hereafter for $D_{k}(\alpha)$. We observe that $\rho_{4}, \rho_{-4}$ are independent of $\tau_{1}$ and $\tau_{2}$. For (2.05)

$$
\begin{aligned}
\rho_{2} & =\frac{1}{2}, \quad \rho_{-2, n+2 p-2}=-\left(\begin{array}{c}
n+2 p \\
2
\end{array}\right) ; \\
\rho_{0, n+2 p} & =\left[-\frac{1}{2}(n+2 p)^{2}-\frac{1}{2}(n+2 p)-\frac{1}{4}\right] .
\end{aligned}
$$

For (2.06)

$$
\rho_{2}=-\frac{1}{2}, \quad \rho_{-2, n+2 p-2}=\left(\begin{array}{c}
n+2 p \\
2
\end{array}\right),
$$

and $\rho_{0, n+2 p}$ is the same as in (2.12). Moreover $\rho_{2}$ and $\rho_{-2}$ are of the same magnitude but opposite in sign in (2.12) and (2.13), while the coefficients $\rho_{0}, \rho_{4}, \rho_{-\rightarrow}$ are the same in both equations. It is this fact that will lead, by induction, to (1.07) and (1.08).

When (2.09) is substituted into (2.071) and use is made of (2.10)-(2.13), one obtains, for the terms of $Y_{1}$ :

$$
\begin{aligned}
\sum_{p=-2}^{p=2}- & 2 p c_{n+2 p, 1} D_{n+2 p} \\
& =\left(\rho_{0, n}+d_{1}\right) D_{n}+\rho_{2} D_{n+2}+\rho_{-2, n-2} D_{n-2}+\rho_{4} D_{n+4}+\rho_{-4, n-4} D_{n-4} .
\end{aligned}
$$

The above must hold for arbitrary values of $\alpha$; hence the coefficients of $D_{k}$ on the right and left sides of (2.14) must be equated to one another. Because 
the coefficient of $D_{n}$ vanishes on the left-hand side of (2.14), one obtains $d_{1}=-\rho_{0, n}$, and so the first coefficient in the asymptotic expansion of $A$ has been determined. Moreover, for (2.05), $Y_{1}=Z_{0,1}+Z_{1,1}$; for (2.06), $Y_{1}=Z_{0,1}$ $-Z_{1,1}$ where

$$
\begin{aligned}
Z_{0,1} & =c_{n+4,1} D_{n+4}+c_{n-4,1} D_{n-4} ; \quad Z_{1,1}=c_{n+2,1} D_{n+2}+c_{n-2,1} D_{n-2}, \\
c_{n+2 p, 1} & =-\rho_{2 p, n+2 p} / 2 p, \text { with the values of } \rho_{2 p} \text { of Case } 1, c_{n, j}=0, j \neq 0 .
\end{aligned}
$$

Assume that for $j \leqq m-1, Y_{j}=Z_{0, j}+Z_{1, j}$ in case of (2.05), and $Y_{j}=Z_{0, j}$ $-Z_{1, j}$ in case of (2.06). Further assume that the $d_{j}$ are the same in both cases, up to $j=m-1$. To obtain the functions entering into $Y_{m}$, one has the following explicit relations:

$$
\sum_{p=-2 m}^{p=2 m}\left(-2 p c_{n+2 p, m} D_{n+2 p}\right)=Q_{0}+Q_{1} ;
$$

where

$$
\begin{aligned}
& Q_{0}=\sum_{j=0}^{j=m-1} d_{m-j}\left[\sum_{p=-m}^{p=m} c_{n+4 p, j} D_{n+4 p}\right]+\sum_{p=-m}^{p=m} f_{4 p, m-1} D_{n+4 p}, \\
& Q_{1}=\sum_{j=0}^{j=m-1} d_{m-j}\left[\sum_{p=-m}^{p=m} c_{n+4 p+2 j} D_{n+4 p+2}\right]+\sum_{p=-m}^{p=m} f_{4 p+2, m-1} D_{n+4 p+2},
\end{aligned}
$$

with

$$
\begin{aligned}
f_{4 p, m-1}= & \rho_{4} c_{n+4 p-4, m-1}+\rho_{2} c_{n+4 p-2, n-1}+\rho_{-2, n+4 p} c_{n+4 p+2, m-1} \\
& +\rho_{0, n+4 p} c_{n+4 p, m-1}+\rho_{-4, n+4 p} c_{n+4 p+4, m-1} \\
f_{4 p+2, m-1}= & \rho_{4} c_{n+4 p-2, m-1}+\rho_{2} c_{n+4 p, m-1}+\rho_{0, n+4 p+2} c_{n+4 p+2, m-1} \\
& +\rho_{-2, n+4 p+2} c_{n+4 p+4, m-1}+\rho_{-4, n+4 p+2} c_{n+4 p+6, m-1}
\end{aligned}
$$

with the convention that $c_{n+2 r, j}=0$ if $|r|>2 j$.

Making use of the fact that $\rho_{2}$ and $\rho_{-2}$ change sign for (2.06), as compared with (2.05), and that for all $j \leqq n-1$ the $c_{n+4 p+2}$ are also of opposite sign in the two cases, it follows that all the $f_{4 p, m-1}$ are the same in cases (2.05) and (2.06), while all the $f_{4 p+2, m-1}$ are of opposite sign. Hence the induction to establish (1.08) can be easily completed, provided we can show that $d_{m}$ is the same for both cases. The condition determining $d_{m}$ is that the coefficient of $D_{n}$ on the right hand sides of (2.15) and (2.16) must vanish. Since $D_{n}$ enters only into (2.15), which is the same in the two cases, it follows that $d_{m}$ is also the same. Hence $Q_{0}$ can be identified with $Z_{0, m}$ and $Q_{1}$ with $Z_{1, m}$. The fact that (1.08) is an asymptotic expansion for large $q$, with the appropriate properties at $x=\pi / 2$, follows from the proof in [3], since (1.08) is of the same form as (1.05). The factor $S_{n}$ depends only on the normalization required.

The differential equation (2.00) of course has two linearly independent solutions, and $\alpha=0$ is a regular point of the equation. The functions $D_{n+2 p}$ 
satisfy (2.10). The latter equation also admits of a second, linearly independent solution $F_{m}$ which can be taken as an odd function of $\alpha$ when $D_{n}$ is even and as an even function of $\alpha$ when $D_{n}$ is odd. There will be recurrence relations among the $F_{m}$ similar to those among the $D_{n+2 p}$, and with very minor changes, the algorithm (2.07)-(2.08) will yield a second, linearly independent asymptotic solution, which will be valid for sufficiently small values of $\alpha$ at least. When the two linearly independent solutions are considered together, it is clear that any function that has the same parity as $D_{n}$ and satisfies (2.00) will have an asymptotic expansion that is proportional to (1.07), and has no part of the solutions involving the $F_{m}$. This fact can be summarized in the following lemma:

Lemma 1. If $M(\alpha)$ and $N(\alpha)$ both satisfy (2.00) and if the asymptotic expansions of both functions are of the form (1.01), then $M(\alpha)$ is proportional to $N(\alpha)$, up to an $O q^{(-1 / 2)(i+1)}$.

Now consider the function $y$ defined by (2.02). Clearly it satisfies (2.00), as a function of $\alpha$. Also, if $0<c<x<\pi-c<\pi$, then

$$
\sin x=\left(1-\frac{\alpha^{2}}{u}\right)^{1 / 2}, \quad u=4 q^{1 / 2} .
$$

In this range it is permissible to expand the above radical as a function of $\alpha^{2}$. After this is done, and the resulting terms are multiplied by $U(\alpha)$, let us use the well known relation

$$
\alpha^{2} D_{m}(\alpha)=(2 m+1) D_{m}(\alpha)+D_{m+2}(\alpha)+m(m-1) D_{m-2}(\alpha),
$$

and so convert the various terms involving powers of $\alpha^{2 k}$ to terms of the form $D_{n+2 p}(\alpha)$. It can be easily verified by direct comparison that the resulting function agrees to terms in $1 / q$ with the corresponding terms of (1.07). Since $\left.{ }^{2}\right)$ both (1.07) and (1.08) satisfy (2.00) to within $O\left(q^{(-1 / 2)(j+1)}\right)$, it follows from Lemma 1 that the two expansions are the same to that accuracy. This analysis therefore provides justification for the use of (1.07) as an approximation to $s e_{n+1}(x, q)$ for the range between 0 and $\pi$. Equation (1.08), on the other hand, holds for all real values of $x$. Aside from this rather minor advantage, (1.08) is preferable to (1.07) over certain regions. This is so because in practice only two (or at most three) terms of the developments (1.07) and (1.08) can be computed. When the expansions are truncated after two terms, there may be considerable difference between (1.07) and (1.08), with the latter usually better for the smaller angles and orders greater than 2 . The examples in $\$ 3$ exhibit this.

The solution (1.06) differs from the correct approximations in the order $q^{-1 / 2}$, no matter how many terms are carried in the expansion.

(2) It would have been enough to show that agreement is obtained in the constant term. The verification to terms in $1 / q$ confirms the accuracy of corresponding terms in $S_{n}$. 
3. Explicit formulas and examples. The new results are given here along with those that have previously appeared so that $S_{n}$ may be examined in the context of the related functions of even order. Formulas (3.02)-(3.07) contain the term in $q^{-3 / 2}$ which has not heretofore appeared in the literature. In addition, the term involving $q^{-3 / 2}$ has been added to the normalization factors $C_{n}$ and $S_{n}$, for general values of $n$. This term arises, in fact, from terms in $Z_{0, j}$ and $Z_{1, j}$ for $j \leqq 2$. It might therefore be argued that the power $q^{-3 / 2}$ should be used in the normalization factors, even though $Z_{0, j}$ and $Z_{1, j}$ stop with terms in $1 / q$. However, one does not necessarily improve results that way. The extent to which $q^{-3 / 2}$ of the normalization factors affect the calculations may be regarded as a measure of the uncertainty of the value, if $Z_{0, j}$ and $Z_{1, j}$ are carried to $j=2$ only.

Included in the formulas are values of $c e_{2 n}(0, q) / c e_{2 n}(\pi / 2, q)$ and similar functions, for low orders. These are very useful for calculating the normalization factors applicable to Goldstein's formulas [1], in regions where (1.07) cannot be used. All the results in [7], where these ratios originate, were checked; only Sips (34) required change in the last coefficient, and it is believed that the comparable formula (3.17) below is correct. These ratios are given here because a few misprints, some of which originate in [6], may lead an unwary reader astray. To anyone who has tried to derive some of the very useful results Sips has given, the wonder is that the errors in his calculations are so few!

The writer is indebted to Donald Clemm of the Aeronautical Research Laboratory for help with the many calculations that were involved in the results given here. He checked most of the formulas and provided all the examples. Martha Elmore assisted with some of the computations required in Goldstein's formula.

\section{FORMULAS}

$$
\begin{aligned}
c e_{n}(x, q) & =C_{n}\left[Z_{0, n}(\alpha)+Z_{1, n}(\alpha)\right] . \\
s e_{n+1}(x, q) & =S_{n}\left[Z_{0, n}(\alpha)-Z_{1, n}(\alpha)\right] \sin x .
\end{aligned}
$$

In the following the symbol $D_{k}=D_{k}(\alpha), \alpha=2 q^{1 / 4} \cos x$. For $n=0$,

$$
\begin{aligned}
Z_{0,0}= & D_{0}-\frac{D_{4}}{64 q^{1 / 2}}+\frac{1}{16 q}\left[\frac{-D_{4}}{8}+\frac{D_{8}}{512}\right] \\
& +\frac{1}{64 q^{3 / 2}}\left[\frac{-99 D_{4}}{256}+\frac{3 D_{8}}{256}-\frac{D_{12}}{24576}\right]+\cdots ; \\
Z_{1,0}= & \frac{-D_{2}}{16 q^{1 / 2}}+\frac{1}{16 q}\left[\frac{-9 D_{2}}{16}+\frac{D_{6}}{64}\right] \\
& +\frac{1}{64 q^{3 / 2}}\left[\frac{-61 D_{2}}{32}+\frac{25 D_{6}}{256}-\frac{5 D_{10}}{10240}\right]+\cdots ;
\end{aligned}
$$


(3.04) $\quad C_{0}=(\pi / 2)^{1 / 4} q^{1 / 8}\left[1+\frac{1}{8 q^{1 / 2}}+\frac{108}{2048 q}+\frac{572}{16384 q^{8 / 2}}+\cdots\right]^{-1 / 2}$;

(3.05) $\quad S_{0}=(\pi / 2)^{1 / 4} q^{1 / 8}\left[1-\frac{1}{8 q^{1 / 2}}-\frac{84}{2048 q}-\frac{476}{16384 q^{3 / 2}}+\cdots\right]^{-1 / 2}$

For $n=1$,

$$
\begin{aligned}
Z_{0,1}= & D_{1}-\frac{D_{5}}{64 q^{1 / 2}}+\frac{1}{16 q}\left[\frac{-3 D_{5}}{16}+\frac{D_{9}}{512}\right] \\
& +\frac{1}{64 q^{3 / 2}}\left[\frac{-207 D_{5}}{256}+\frac{D_{9}}{64}-\frac{D_{13}}{24576}\right]+\cdots ;
\end{aligned}
$$

$$
\begin{aligned}
Z_{1,1}= & -\frac{D_{3}}{16 q^{1 / 2}}+\frac{1}{16 q}\left[\frac{-15 D_{3}}{16}+\frac{D_{7}}{64}\right] \\
& +\frac{1}{64 q^{3 / 2}}\left[\frac{-153 D_{3}}{32}+\frac{35 D_{7}}{256}-\frac{D_{11}}{2048}\right]+\cdots ;
\end{aligned}
$$

$$
C_{1}=(\pi / 2)^{1 / 4} q^{1 / 8}\left[1+\frac{3}{8 q^{1 / 2}}+\frac{159}{512 q}+\frac{1389}{4096 q^{3 / 2}}+\cdots\right]^{-1 / 2} \text {; }
$$

$$
S_{1}=(\pi / 2)^{1 / 4} q^{1 / 8}\left[1-\frac{3}{8 q^{1 / 2}}-\frac{81}{512 q}-\frac{669}{4096 q^{3 / 2}}\right]^{-1 / 2}
$$

For general $n$ :

$$
\begin{aligned}
Z_{0, n}= & D_{n}+\frac{1}{4 q^{1 / 2}}\left[\frac{-D_{n+4}}{16}+\frac{3}{2}\left(\begin{array}{l}
n \\
4
\end{array}\right) D_{n-4}\right] \\
+ & \frac{1}{16 q}\left[\frac{D_{n+8}}{512}-\frac{(n+2) D_{n+4}}{16}+\frac{3}{2}(n-1)\left(\begin{array}{l}
n \\
4
\end{array}\right) D_{n-4}\right. \\
& \left.+\frac{315}{4}\left(\begin{array}{l}
n \\
8
\end{array}\right) D_{n-8}\right]+\cdots ;
\end{aligned}
$$

$$
\begin{aligned}
Z_{1, n}= & \frac{1}{4 q^{1 / 2}}\left[-\frac{1}{4} D_{n+2}-\frac{n(n-1)}{4} D_{n-2}\right] \\
& +\frac{1}{16 q}\left[\frac{D_{n+6}}{64}+\frac{n^{2}-25 n-36}{64} D_{n+2}\right. \\
& \left.+\frac{n(n-1)\left(-n^{2}-27 n+10\right) D_{n-2}}{64}-\frac{45}{4}\left(\begin{array}{l}
n \\
6
\end{array}\right) D_{n-6}\right]+\cdots ;
\end{aligned}
$$

$$
C_{n}=\left[(\pi / 2)^{1 / 4} q^{1 / 8} /(n !)^{1 / 2}\right] U_{0} ; \quad S_{n}=\left[(\pi / 2)^{1 / 4} q^{1 / 8} /(n !)^{1 / 2}\right] V_{0} ;
$$


(3.13)

$$
\begin{aligned}
U_{0}= & {\left[1+\frac{2 n+1}{8 q^{1 / 2}}+\frac{n^{4}+2 n^{3}+263 n^{2}+262 n+108}{2048 q}\right.} \\
& \left.+\frac{6 n^{5}+15 n^{4}+1280 n^{8}+1905 n^{2}+1778 n+572}{16384 q^{3 / 2}}\right]^{-1 / 2}+\cdots ;
\end{aligned}
$$

$$
\begin{aligned}
V_{0}= & {\left[1-\frac{2 n+1}{8 q^{1 / 2}}+\frac{n^{4}+2 n^{3}-121 n^{2}-122 n-84}{2048 q}\right.} \\
& \left.+\frac{2 n^{5}+5 n^{4}-416 n^{8}-629 n^{2}-1162 n-476}{16384 q^{8 / 2}}+\cdots\right]^{-1 / 2} ;
\end{aligned}
$$

(3.14) $\left[c e_{0}(0, q)\right] /\left[c e_{0}(\pi / 2, q)\right]$

$$
=2(2)^{1 / 2} e^{-2(q)^{1 / 2}}\left(1+\frac{1}{16(q)^{1 / 2}}+\frac{9}{256 q}+\cdots\right) ;
$$

(3.15) $\left[c e_{2}(0, q)\right] /\left[c e_{2}(\pi / 2, q)\right]$

$$
=-32(2 q)^{1 / 2} e^{-2(q)^{1 / 2}}\left(1-\frac{1}{16(q)^{1 / 2}}+\frac{29}{128 q}+\cdots\right) ;
$$

(3.16) $\quad\left[c e_{1}(0, q)\right] /\left[c e_{1}^{\prime}(\pi / 2, q)\right]$

$$
=-4(2)^{1 / 2} e^{-2(q)^{1 / 2}}\left(1+\frac{3}{16(q)^{1 / 2}}+\frac{45}{256 q}+\cdots\right) ;
$$

(3.17) $\quad\left[c e_{3}(0, q)\right] /\left[c e_{3}^{\prime}(\pi / 2, q)\right]$

$$
=\frac{64}{3}(2 q)^{1 / 2} e^{-2(q)^{1 / 2}}\left(1-\frac{3}{16(q)^{1 / 2}}+\frac{47}{128 q}+\cdots\right) ;
$$

(3.18) $\quad\left[s e_{1}^{\prime}(0, q)\right] /\left[s e_{1}(\pi / 2, q)\right]$

$$
=4(2 q)^{1 / 2} e^{-2(q)^{1 / 2}}\left(1-\frac{3}{16(q)^{1 / 2}}-\frac{11}{256 q}+\cdots\right) ;
$$

(3.19) $\quad\left[s e_{3}^{\prime}(0, q)\right] /\left[\operatorname{se}_{3}(\pi / 2, q)\right]$

$$
=-64(2)^{1 / 2} q e^{-2(q)^{1 / 2}}\left(1-\frac{21}{16(q)^{1 / 2}}-\frac{17}{128 q}+\cdots\right) ;
$$

(3.20) $\left[s e_{2}^{\prime}(0, q)\right] /\left[\operatorname{se}_{2}^{\prime}(\pi / 2, q)\right]$

$$
=-8(2 q)^{1 / 2} e^{-2(q)^{1 / 2}}\left(1-\frac{9}{16(q)^{1 / 2}}-\frac{39}{256 q}+\cdots\right)
$$

(3.21) $\left[\operatorname{seq}_{4}^{\prime}(0, q)\right] /\left[\operatorname{se}_{4}^{\prime}(\pi / 2, q)\right]$

$$
=\frac{128}{3}(2)^{1 / 2} q e^{-2(q)^{1 / 2}}\left(1-\frac{31}{16(q)^{1 / 2}}-\frac{15}{128 q}+\cdots\right) .
$$


Examples for $80^{\circ}, 60^{\circ}$, and $30^{\circ}$

$\mathbf{q}=\mathbf{3 6}$

\begin{tabular}{|c|c|c|c|c|c|c|c|}
\hline \multicolumn{5}{|c|}{$c e_{n}(x, q)$} & \multicolumn{3}{|c|}{$\operatorname{se}_{n+1}(x, q)$} \\
\hline$n$ & $x^{0}$ & $\begin{array}{c}\text { By (3.00) } \\
-(3.13)\end{array}$ & $\begin{array}{l}\text { By Goldst. } \\
\text { Formula(4) }\end{array}$ & $\begin{array}{l}\text { Correct } \\
\text { Value }\end{array}$ & $\begin{array}{c}\text { By (3.00) } \\
-(3.13)\end{array}$ & $\begin{array}{l}\text { By Goldst. } \\
\text { Formula(4) }\end{array}$ & $\begin{array}{l}\text { Correct } \\
\text { Value }\end{array}$ \\
\hline \multirow[t]{3}{*}{0} & 80 & 1.45392 & 1.4249 & 1.4538713 & 1.4539 & 1.4252 & 1.4538713 \\
\hline & 60 & .36116 & .35355 & .3607440 & .36006 & .35364 & .36074400 \\
\hline & 30 & .00470 & .00489 & .0049928 & .005271 & .004894 & .00499268 \\
\hline \multirow[t]{4}{*}{1} & 80 & 1.20522 & 1.2290 & 1.2044061 & 1.2049 & 1.2317 & 1.2044062 \\
\hline & 80 & $1.204566\left({ }^{3}\right)$ & & & $1.20448\left({ }^{3}\right)$ & & \\
\hline & 60 & $\begin{array}{l}.91749 \\
.91683\left(^{(8)}\right.\end{array}$ & .93535 & .9166781 & $\begin{array}{l}.91406 \\
\left..91613()^{3}\right)\end{array}$ & .9374 & .9166782 \\
\hline & 30 & .02882 & .02807 & .0275131 & .02900 & .02812 & .0275112 \\
\hline \multirow[t]{3}{*}{2} & 80 & -.31080 & -.31069 & -.3106320 & -.3112 & -.31498 & -.3106322 \\
\hline & 60 & 1.3427 & 1.4267 & 1.3403248 & 1.3381 & 1.4401 & 1.3403284 \\
\hline & 30 & .1111 & .10631 & .1002516 & .1026 & .10728 & .1002262 \\
\hline \multirow[t]{3}{*}{3} & 80 & -1.0857 & -1.0410 & -1.076387 & -1.0816 & -1.2504 & -1.0767231 \\
\hline & 60 & 1.1049 & 1.2557 & 1.0943113 & 1.1008 & 1.2868 & 1.0944138 \\
\hline & 30 & .3079 & .3042 & .2749215 & .2683 & .31146 & .2746626 \\
\hline
\end{tabular}

(8) Using terms involving $q^{-\mathbf{3} / \mathbf{2}}$.

(4) Based on formula (4.08), (4.09) of [4, p. xxvi], with normalization factors determined from $c e_{n}(0, q)$ or $\operatorname{se}_{n}^{\prime}(0, q)$ by $(3.14)-(3.21)$ of this paper.

\section{REFERENCES} 336.

1. S. Goldstein, Mathieu functions, Cambridge Philos. Soc. Trans. vol. 23 (1927) pp. 303-

2. E. L. Ince, Tables of elliptic cylinder functions, Proc. Roy. Soc. Edinburgh vol. 52 (1932) pp. $355-423$.

3. J. Meixner and F. W. Schäfke, Mathieusche Funktionen und Sphäroidfunktionen, \$82.331-2.333, Berlin, Springer, 1954.

4. National Bureau of Standards, Tables relating to Mathieu functions, New York, Columbia University Press, 1951.

5. Robert Sips, Représentation asymptotique des fonctions de Mathieu et des fonctions d'onde spheroidales, Trans. Amer. Math. Soc. vol. 66 (1949) pp. 93-134.

6. - Les constantes caracteristiques de l'équation intégrale des fonctions de Mathieu, Bull. Soc. Roy. Sci. Liège vol. 21 (1953) pp. 141-157.

7. - Représentation asymptotique des fonctions de Mathieu et des fonctions sphéroidales. II, Trans. Amer. Math. Soc. vol. 90 (1959) pp. 340-368.

Aeronautical Research Laboratory, Wright-Patterson Air Force Base, Ohio 